\title{
Fabrication of Mirror Image Prosthetic Ears-A Short Review
}

\section{Suman Thotapalli*}

Lecturer, Division of Prosthodontics, Riyadh Colleges of Dentistry and Pharmacy, Riyadh, Saudi Arabia

\begin{abstract}
Maxillofacial prosthodontics gains utmost significance as the process not only tries to develop acceptable esthetics but also tries to enhance the psychological comfort of the patient by restoring the defective or missing craniofacial structures. This science has developed into a more reliable and predictable process due to ever increasing development of materials and equipments used in such procedures, but no single procedure stands out as the most effective method in all the situations. The present article tries to give an overview of different methods used to fabricate auricular prosthesis so as to give an array options available to arrive at the best possible outcome.
\end{abstract}

Keywords: Auricular prosthesis; Maxillo-facial prosthesis; Rapid prototype

\section{Introduction}

The fabrication of any extra oral maxillofacial prosthesis presents the prosthodontist with several phenomenal challenges. Foremost among them is the flawless recreation of the same anatomic form that was present preoperatively. The Challenge of recreating preoperative anatomic contour is sometimes complicated by the absence of a preoperative record. This is common when the loss is congenital or unexpected as with the loss of human ear. In these conditions the preoperative bilaterally symmetry can be recreated using mirror image modeling. The present article attempts to review different techniques reported in literature for mirror image modeling in the fabrication of auricular Prosthesis where preoperative records are not available.

\section{Various Techniques for Fabrication of Auricular Prosthesis}

\section{Conventional technique}

Conventionally if pre-operative casts are not available. The prosthesis can be sculpted from the beginning. Taking into consideration the complex anatomy of the ear this procedure is laborious, time consuming and totally depends on the artistic skill of the operator. This task is facilitated by sectioning cast of the normal side into equal divisions so that contours are more easily verified [1].

Alternately the donor technique can be employed. This is an easier technique. A relative/family member with ear contours that closely match with that of the patient is selected. Impression of ear is obtained and wax cast is retrieved. The wax cast is adapted and re-contoured as necessary. Image editing software such as Adobe Photoshop can be used to obtain mirror image of normal ear; this image can be used as an aid in contouring the wax pattern for the prosthesis (Figure 1).

Shaper/Tracer: A mirror image pantograph can be used to obtain cast of the opposite ear. The machine uses a metal shaper activated by hand which consists of arm bearing cutting and tracing tools which move in opposite directions as the master cast is moved in horizontal plane. The machine contains hand activated metal shaper. The machine consists of two articulated arms with tracing or cutting tools which move in an opposing or parallel direction as the master model is traversed in the horizontal plane [2].

The photocopy technique: The photocopy technique of obtaining sculpted cast by overlapping color slides. A Photocopy of the cast of the normal ear is acquired using a transparent sheet. This sheet is pasted on to a glass plate. The glass plate is inverted and superimposed on to the cast of the defective side and is used as an aid in molding the wax pattern.

Non-contact 3-Dimensional (3-D) models of the facial region are made possible with advances in the digital imaging technology. The various steps involved in the fabrication of such prosthesis are:

\section{Acquisition of 3-D data using scanning.}
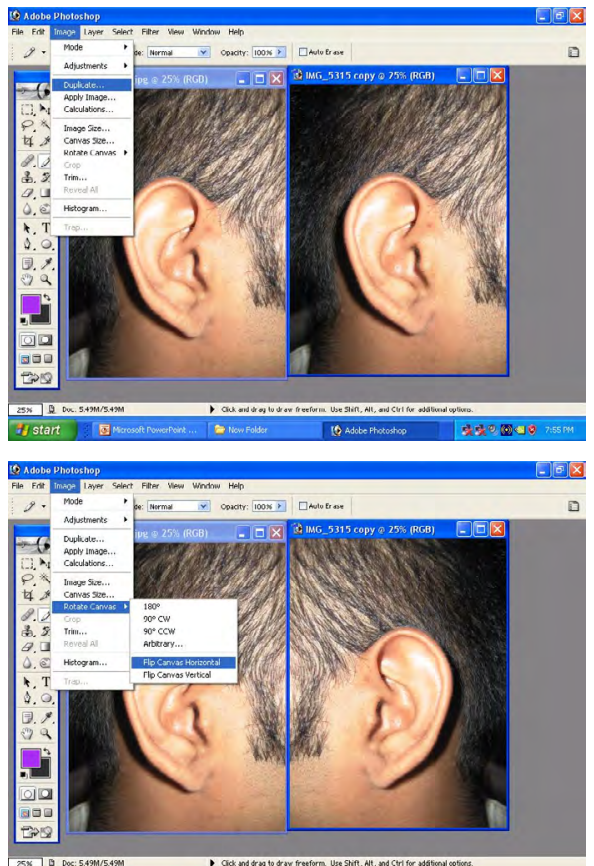

Figure 1: Image editing using Adobe Photoshop.

*Corresponding author: Suman Thotapalli, Lecturer, Division of prosthodontics, Riyadh colleges of dentistry and pharmacy, Riyadh, Saudi Arabia, Tel: +966544868463; E-mail: thotapallimds08@gmail.com

Received July 04, 2013; Accepted October 24, 2013; Published October 28, 2013

Citation: Thotapalli S (2013) Fabrication of Mirror Image Prosthetic Ears-A Short Review. Anaplastology 2: 120. doi: 10.4172/2161-1173.1000120

Copyright: $\odot 2013$ Thotapalli S. This is an open-access article distributed under the terms of the Creative Commons Attribution License, which permits unrestricted use, distribution, and reproduction in any medium, provided the original author and source are credited. 
2. Generation of blue print of the defect area.

\section{Obtaining a prototype.}

Computerized tomography (CT) scanning: CT scan acquires data by recording in slices which can be used to reconstruct anatomical structures or fabricate prosthesis with accuracy and details. The wide spread use of CT scan also casts a shadow on the application as it may expose the patient to high radiation dosage.

Techniques to obtain 3-dimensional (3-D) models using data obtained from CT scan and milled using polyurethane blocks to obtain ideal soft tissue models for waxing have been developed in recent times [3].

Magnetic Resonance Imaging (MRI): This proves a viable alternative as the exposure to radiation is substantially reduced in this imaging technique. The disadvantage being its application in situations with many metallic restorations leading to scattering and artifact formation. One another disadvantage being the necessity of patient to remain still during the entire length of procedure.

3-D laser scanning system: Laser scanning has the disadvantage of inability to record the details in the undercut areas as the laser can be directed only vertically. Such problems are corrected with the advent of 3-D laser scanning technique [4].

A technique of obtaining a scan from cast of existing ear using 3-D laser scanner, creating a mirror image of the scan to obtain a rapid prototype model of the deficient side using resin material. A 3D laser scanner develops an integrated 3D digital image of the unaffected ear, which is copied and then mirrored. A rapid prototyping machine collects the necessary data to manufacture the definitive resin ear $[5,6]$.

\section{Steps in laser scanning:}

- Acquisition of "emodel" (3-D dental cast) using laser scanning.

- Utilizing color pins to guide the location of the cast.

- Positioning cast in 8 different locations to record the undercut areas.

- Over one million points are recorded to obtain scan of the cast which are used to generate emodel software data.

- Produce 3-D mirror image of the cast (Figure 2).

Computer Numerically Controlled (CNC) milling: This encompasses data acquisition using laser scanning and software to collect data to form reverse of the normal ear. This data is fed into a cutter which mills blocks of polytetraflouroethylene into the desired shape. The disadvantages being inability to mill the areas which are in an undercut that are not recorded by the scanner, a maxillofacial technician then has to modify the contours to obtain the desired shape. The disadvantage of inability in reproducing undercut areas can be overcome by techniques like rapid prototyping [7].

Rapid prototyping: It forms the mold of desired shape by
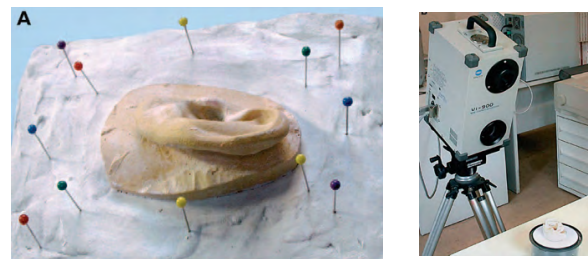

Figure 2: 3-D laser scanning system.
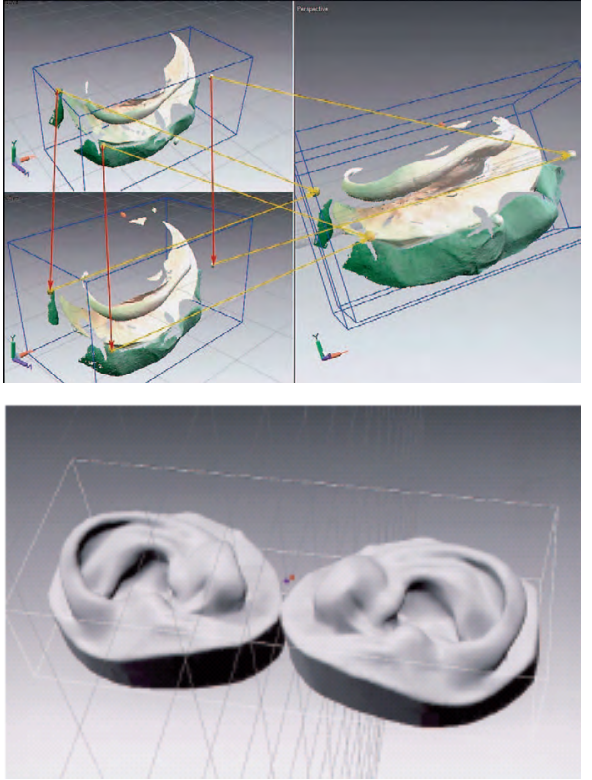

Figure 3: Rapid Prototyping.

deposition of material into layers rather than by milling from a single block which has an added advantage of reproduction of undercuts and finer details, such details were not possible with previous generation of equipments. This also adds to the merits of reduction in the wastage of material as the material is layered in the designated areas minimizing the wastage [8,9] (Figure 3).

Stereolithography: This method generally uses UV (Ultraviolet) light to cure resin to form a desired shape. The currently available variants of this technique in the market allows to create a model with thickness to as less as $0.5 \mathrm{~mm}$. They can use UV light to cure resin, fuse layers of metal/resin and can laminate thin sheets to obtain the desired shape [10].

\section{Conclusion}

Prosthesis made by CAD/CAM techniques are better than those fabricated by conventional methods. But unfortunately taking into account the complexity and the high cost of the equipment needed, these techniques can only be used in well developed establishments and an academic institution which makes us rely on more conventional techniques for the fabrication of extra-oral maxillofacial prosthesis.

\section{References}

1. Reisberg DJ, Habakuk SW (1990) Nasal conformer to restore facial contour. J Prosthet Dent 64: 699-701.

2. Beumer J, Curtis TA, Marunick MT (1996) Maxillofacial rehabilitation Prosthodontic and Surgical Considerations (2ndedn), St Louis: Ishiyaku Euroamerica.

3. Gurbuz A, Kalkan M, Ozturk AN, Eskitascioglu G (2004) Nasal prosthesis rehabilitation: a case report. Quintessence Int 35: 655-656.

4. Godoy AJ, Lemon JC, Nakamura SH, King GE (1992) A shade guide for acrylic resin facial prostheses. J Prosthet Dent 68: 120-122.

5. Wolfaardt JF, Wilkes GH, Parel SM, Tjellström A (1993) Craniofacial osseointegration: the Canadian experience. Int J Oral Maxillofac Implants 8: 197-204.

6. Tolman DE, Desjardins RP (1991) Extraoral application of osseointegrated implants. J Oral Maxillofac Surg 49: 33-45. 
7. Parel SM, Tjellström A (1991) The United States and Swedish experience with osseointegration and facial prostheses. Int J Oral Maxillofac Implants 6: 75-79.

8. Cheng AC, Morrison D, Cho RS, Archibald D (1998) Vacuum-formed matrix as a guide for the fabrication of craniofacial implant tissue bar-retained auricular prostheses. J Prosthet Dent 79: 711-714.
9. McKinstry RE (1995) Fundamentals of facial prosthetics, (1stedn) Arlington $A B I$ Professional Publications.

10. Wolfaardt JF, Coss $P$ (1996) An impression and cast construction technique for implant-retained auricular prostheses. J Prosthet Dent 75: 45-49. 